\title{
ON THE EXISTENCE OF LOCI WITH GIVEN SINGULARITIES*
}

\author{
BY \\ SOLOMON LEFSCHETZ
}

Index

81. Introduction. ......................................... 23

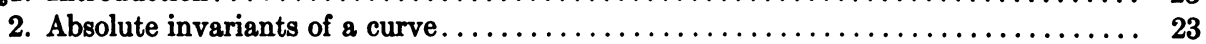

3. Conditions for a double point . . . . . . . . . . . . .

5. The maximum number of cusps $\ldots \ldots \ldots \ldots \ldots \ldots \ldots \ldots \ldots \ldots \ldots \ldots \ldots \ldots \ldots \ldots, 27$

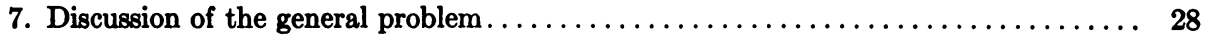

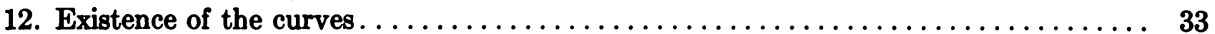

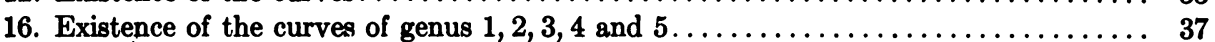

20. Singularities of surfaces and higher varieties....................... 39

Introduction.

1. The numerical relations existing between ordinary or so-called Plïckerian singularities of a plane curve were determined as early as 1834 by Plücker, but the inverse question has been left almost untouched. It may be stated thus: To show the existence of a curve having assigned Plückerian characters; and is equivalent to the determination of the maximum of cusps $\kappa_{M}$ that a curve of order $m$ and genus $p$ may have. Veronese $\dagger$ has solved the question for rational curves. As an example of errors that have been committed in this direction, we cite SALMON-FIEDLER's $\ddagger$ statement that a seventhic may have 13 cusps, in which case however it is found that it would have $(-2)$ bitangents!

In this paper a condition derived from the theory of invariants is given for the characteristics of a curve, a discussion of the a priori possible curves follows, and the existence of the curves within a certain range is then shown.

Finally a few words will be said as to the extension of the theory to surfaces and higher varieties.

\section{Absolute invariants of a curve.}

2. Let $e_{1}, e_{2}, \cdots, e_{R}$ be the coefficients of a quantic $Q$ in $x_{1}, x_{2}, x_{3}$, and $e_{1}^{\prime}, e_{2}^{\prime}, \cdots, e_{R}^{\prime}$ be the coefficients of $Q^{\prime}$, a quantic in $\left(x_{1}^{\prime}, x_{2}^{\prime}, x_{3}^{\prime}\right)$ obtained from

* Presented to the Society, September 12, 1911.

†Uber die Methode des Projicirens und Schneidens, Mathematis che Annalen, vol. 19 (1882), p. 209.

$\ddagger$ Höhere Ebene Kurven, p. 83. 
$Q$ by the transformation:

$$
x_{i}=\sum_{k=1}^{3} \lambda_{i k} x_{k}^{\prime}
$$

The equations of transformation are:

$$
e_{j}^{\prime}=f_{j}(e, \lambda)
$$$$
(j=1,2, \cdots, R)
$$

linear in the $(e)$ 's and rational in the $\lambda$ 's, with no other denominator than some power of the determinant $\Delta$ of the transformation which we will suppose not to vanish. Following ANDOYER * let us consider the array:

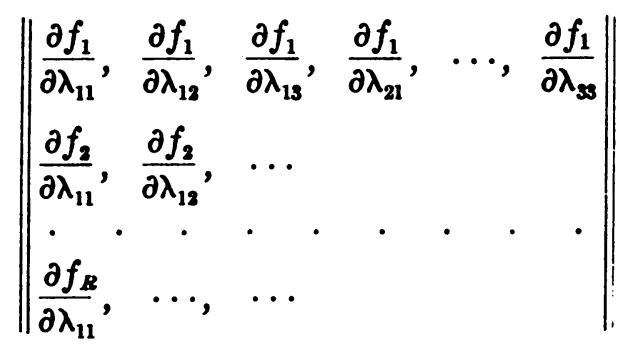

and suppose that at least one of the determinants of order $r(r<9)$ that can be derived from it does not vanish, while all those of order $r+1$ do. Then the system can be reduced to an equivalent one formed of $r$ equations containing the $(\lambda)$ 's, and $(R-r)$ freed from them. $\dagger$ The latter will have to be satisfied by the coefficients of any quantic derived from $Q$ by a linear transformation of non-vanishing determinant. The left members of these $(R-r)$ equations will be absolute invariants of $Q$. If $\left(e^{\prime}\right)$ and $\left(e^{\prime \prime}\right)$ are any two quantics, the necessary and sufficient conditions for the passage from one to the other by a linear transformation of non-vanishing $\Delta$, is that

$$
F_{j}\left(e^{\prime}\right)=F_{j}\left(e^{\prime \prime}\right) \quad(j=1,2, \cdots R-r),
$$

the $(F)$ 's being $(R-r)$ algebraically independent absolute invariants. In general

$$
R=\frac{(m+1)(m+2)}{2}
$$

and $r=9$. If $r<9$, then the ( $\lambda$ )'s are functions of $(9-r)$ arbitrary parameters, from which follows at once that $Q$ is invariant under a continuous projective group, hence the curve represented by $Q=0$ is of genus 0 or 1 (Theorem of Schwarz). Hence: $A$ curve of genus greater than one depends upon $\frac{(m+1)(m+2)}{2}-9$ independent absolute invariants. Unless otherwise stated we shall suppose in what follows that $p \geq 2$.

* Lecons sur la theorie des formes, p. 14.

† With poesibly additional equations of restrictive character. 
Conditions for a double point or a cusp.

3. It has been shown by V. SNYDER, ${ }^{*}$ that for a given order $m$, curves exist for each genus up to the maximum $\frac{(m-1)(m-2)}{2}$. From this follows that the system of relations $(E)$ satisfied by the coefficients of the general ternary quantic of order $m$, when its representative curve has $\delta$ double points, cannot be reduced to the system of relations $\left(E^{\prime}\right)$ corresponding to a curve with $(\delta-1)$ double points. Since $\left(E^{\prime}\right)$ is part of $(E)$, it follows that the latter contains at least one more equation than $\left(E^{\prime}\right)$, that is: the addition of a double point increases the number of relations satisfied by the coefficients by at least one unit. If we now consider the most general unicursal curve of order $m$, it can be represented by the system:

$$
x_{i}=\sum_{k=0}^{n} a_{k}^{i} t^{m-k} \quad(i=1,2,3),
$$

which contains $3 m+2$ non-homogeneous arbitraries. But the transformations

$$
t=\frac{\alpha \theta+\beta}{\gamma \theta+1}
$$

are the only ones that leave the curve unchanged, and since this group contains 3 parameters, it follows that there are $\infty^{3 m+2-3}=\infty^{3 m-1}$ rational curves of order $m$. Hence if the general ternary quantic of order $m$ represents one of these curves, its coefficients have to satisfy

$$
\frac{m(m+3)}{2}-(3 m-1)=\frac{(m-1)(m-2)}{2}
$$

relations, which number is precisely the number of double points then imposed upon the curve. Hence, each double point reduces the number of arbitraries by just one unit.

In what precedes we suppose of course that the only conditions imposed refer to the number of double points, and that, for instance, their position is left arbitrary. This arbitrariness however is not always unrestricted. Thus it is not enough to state that a sextic of deficiency 1 has 9 double points, but it must also be said then that these are not on a cubic; for if not so, the latter counted twice would give the sextic required. Such an additional condition however is not expressed by a relation but by an inequality, and furthermore involves the coördinates of the double points in question.

4. Let us now consider the number of relations indicating the presence of $\kappa$ cusps. Since the system $\left(E_{1}\right)$ indicating that there are $\delta$ double points

\footnotetext{
* Construction of curves of given deficiency. Bulletin of the American Mathe-
} matical Society, vol. 15 (1908), p. 3. 
and $\kappa$ cusps of assigned position, has only $\kappa$ more relations than the system $(E)$ corresponding to $(\delta+\kappa)$ double points, certainly when $\kappa$ double points are changed into cusps no more than $\kappa$ relations between the coefficients alone are thereby introduced. Let this number be $\kappa-\lambda, \lambda$ will depend upon $m, p, \kappa$. Whenever we can show that there are curves of order $m_{0}$, genus $p_{0}$, with either $\kappa_{0}$ or $\left(\kappa_{0}+1\right)$ cusps, we shall have

$$
\lambda\left(m_{0}, p_{0}, \kappa_{0}\right)=\lambda\left(m_{0}, p_{0}, \kappa_{0}+1\right),
$$

since the systems of relations for $\kappa_{0}$ and $\kappa_{0}+1$, between the coefficients of the curve, contain a different number of relations. Also $\lambda\left(m_{0}, p_{0}, \kappa_{0}\right)=0$. It follows that if we show that for given $m, p$, the curve can have successively $1,2, \cdots, \kappa_{1}$ cusps, then $\lambda(m, p, \kappa)=0$ for $\kappa<\kappa_{1}$. In a discussion to follow we will admit as a postulate that $\lambda=0$, and we will say then that the postulate of singularities is satisfied. As we just saw, it is equivalent to the admission that when a curve with characters $(m, p)$ can have $\kappa_{1}$ cusps it can also have any number of cusps smaller than $\kappa_{1}$. Of course this is almost evident, but the possibility of a proof is great, and the postulate in question can be accepted temporarily, pending further increase of knowledge in this direction. As we shall see later, this proposition will be verified within quite a wide range. Meanwhile let us show that it is true when $\kappa<m-2$ or that a curve may acquire successively as many as $(m-2)$ cusps. For this purpose consider the equation:

$$
x_{3} \prod_{i=1}^{n-1}\left(x_{1}+\alpha_{i} x_{2}\right)+\varphi_{m}\left(x_{1}, x_{2}\right)=0,
$$

$\varphi_{m}$ being a binary $m$ thic. If we make

$$
\alpha_{1}=\alpha_{2}=\cdots=\alpha_{k-1}=\alpha_{k} \quad(k \leq m-1),
$$

this equation will represent a special rational curve of order $m$ having at $(0,0,1)$ a point of multiplicity $m-1$, at which $k$ of the $m-1$ tangents coincide, which is equivalent to $\frac{(m-1)(m-2)}{2}-(k-1)$ double points and $(k-1)$ cusps. Here clearly every new cusp adds a new relation.

With regard to curves of the type

$$
\sum_{h=k}^{m} x_{3}^{m-h} \varphi_{h}\left(x_{1}, x_{2}\right)=0
$$

it is interesting to remark that when the multiplicity of $(0,0,1)$ is raised from $k$ to $k+1$, we have to satisfy

$$
\frac{(k+1)(k+2)-\not k(k+1)}{2}=k+1
$$


relations, while only $k$ double points have been added. Hence if we increase the multiplicity of a point by $h$ without changing the genus, we decrease the number of arbitraries by $h$.

\section{The maximum number of cusps.}

5. In what follows we shall assume the truth of the postulate of singularities as stated in $\S 4$. According to this postulate an additional cusp imposes two conditions upon the coefficients of the curve. Each of these conditions will be expressed by the vanishing of an invariant, since both are satisfied by any linear transformed of the curve considered. More properly speaking and with reference to $\S 2$, the absolute invariants of the curve will have to satisfy two new relations. Referring then to the same section we infer that a curve of order $m$ cannot have more than $\left[\frac{m(m+3)}{4}-4\right]$ cusps, with the reservation that its genus shall then be greater than one, and this, as will be seen, is actually the case. If $m \equiv 0$ or $1(\bmod 4)$ this number is equal to $\frac{m(m+3)}{4}-4$, and the curves with the maximum number of cusps can have no other double point. If $m \equiv 2$ or $3(\bmod 4)$ this number is equal to $\frac{m(m+3)-2}{4}-4$ and besides the cusps the corresponding curve can have one more double point.

6. The a priori possible curves will evidently have positive Plückerian characters, and we are thus led to discuss Plïcker's equations in order to find the maximum of cusps alone such that $i$ and $\tau$ are positive or zero, $i$ and $\tau$ being the number of inflexions and bitangents. We shall use only the following three formulæ:

$$
\begin{gathered}
n=m(m-1)-2 \delta-3 \kappa, \quad(n=\text { class }) \\
i=3 m(m-2)-6 \delta-8 \kappa, \\
2(\tau-\delta)=(n-m)(n+m-9) .
\end{gathered}
$$

If we suppose $\delta=0$ they become:

$$
\begin{aligned}
n & =m(m-1)-3 \kappa, \\
i & =3 m(m-2)-8 \kappa, \\
2 \tau & =(n-m)(n+m-9) ;
\end{aligned}
$$

from which we deduce:

hence:

$$
\begin{aligned}
n-m & =m(m-2)-3 \kappa, \\
n+m-9 & =m^{2}-9-3 \kappa,
\end{aligned}
$$

$$
2 \tau=[m(m-2)-3 \kappa]\left[m^{2}-9-3 \kappa\right] \text {. }
$$


The first factor on the right is inferior to the second if $m(m-2)<m^{2}-9$, or if $m>4$; and certainly if we suppose, as we shall do, $m \geq 7$. Hence $\tau \geq 0$ if

$$
\kappa ₹\left[\frac{m(m-2)}{3}\right] \text {. }
$$

It is seen at once that when this condition is fulfilled both $i$ and $n$ are positive. Hence a curve has certainly less than $\frac{m(m-2)}{3}$ cusps. We have to compare this last limit to the one found previously. For this purpose we consider

$$
\frac{m(m-2)}{3}-\left(\frac{m(m+3)}{4}-4\right)=\frac{(m-3)(m-14)+6}{12} .
$$

This difference is negative if $m<14$. Hence for $m \geq 14$ we must use the limit found in the preceding paragraph, while for $m<13$ we must use the limit given above.

We have supposed $m \geq 7$, since all the questions considered in this paper have been solved long ago for $m<7$. We may remark however that the quintic with 5 cusps* and the sextic with nine cusps find their place in this discussion. From the above we must not be surprised however that neither the tricuspidal quartic nor the cubic with one cusp satisfy the condition of this section.

\section{Discussion of the general problem.}

7. We shall now discuss the problem stated in the introduction, assuming of course the truth of the postulate of singularities. If we introduce $p$ in them, the formulæ of Plücker used in $\$ 6$ can be written as follows:

$$
\begin{aligned}
n & =m(m-1)-2(\delta+\kappa)-\kappa \\
& =m(m-1)-(m-1)(m-2)+2 p-\kappa \\
& =2(m+p-1)-\kappa, \\
i & =3(m-2) m-6(\delta+\kappa)-2 \kappa=3 m(m-2)-3(m-1)(m-2) \\
& =3(m+2 p-2)-2 \kappa, \quad+6 p-2 \kappa \\
2 \tau & =2 \delta+(n-m)(n+m-9) \\
& =(m-1)(m-2)-2 p-2 \kappa+(m+2 p-2-\kappa)(3 m+2 p-11-\kappa) \\
& =\kappa^{2}-[4(m+p)-11] \kappa+4\left[(m+p)^{2}-5 m-7 p+6\right] \\
& =\varphi(\kappa ; m, p) .
\end{aligned}
$$

* This is Der Pezzo's quintic. See Des Peqzo: Sulla quintica con cinque punti cuspidale, Napoli Rendic onti, series 2, vol. 3 (1889), p. 46. 
Since we must have only positive or zero characteristics we obtain at once the following conditions:

$$
\begin{gathered}
\kappa<2(m+p-1)=\alpha, \\
\kappa<\frac{8}{2}(m+2 p-2)=\beta, \\
0<\varphi(K ; m, p) .
\end{gathered}
$$

The postulate of singularities gives another inequality when $p \geq 2$ :

$$
\delta+2 \kappa<\frac{m(m+3)}{2}-8,
$$

or

and finally:

$$
\frac{(m-1)(m-2)}{2}-p+\kappa<\frac{m(m+3)}{2}-8 \text {, }
$$

$$
\kappa<3 m+p-9=\gamma
$$

If the postulate is not assumed we have to replace (4) by

$$
\kappa<3 m+p-g+\lambda(m, p)=\gamma^{\prime},
$$

but in this case of course the discussion is difficult.

Let $\kappa_{1}$ and $\kappa_{2}$ be the roots of $\varphi(\kappa ; m, p)=0$ considered as a quadratic in $\kappa$. We must compare then the numbers $\alpha, \beta, \gamma, \kappa_{1}$ and $\kappa_{2}$ and in this will consist the discussion. The roots of $\varphi(\kappa ; m, p)$ are real if

$$
[4(m+p)-11]^{2}-16\left[(m+p)^{2}-5 m-7 p+6\right]
$$

or if

$$
=-8 m+24 p+25 \geq 0,
$$

Also

$$
m<3(p+1)
$$

$\kappa_{1} \kappa_{2}=4\left[(m+p)^{2}-5 m-7 p+6\right]$

if $m>2$, since then

$$
=(m+p)(m+p-5)-2(p-3)>0
$$

$$
\begin{aligned}
& m+p-5>p-3, \\
& m+p>2
\end{aligned}
$$

Finally:

$$
\kappa_{1}+\kappa_{2}=4(m+p)-11>0 \text { if } m \geq 3 \text {. }
$$

Hence if $m \geq 3$, the roots of $\varphi(\kappa ; m, p)$ are either positive or imaginary. As to $\alpha, \beta, \gamma$ we have:

$$
\begin{aligned}
& \alpha-\beta=\frac{1}{2} m-(p-1) \geq 0 \text { if } m \geq 2(p-1), \\
& \alpha-\gamma=-m+p+7 \geq 0 \text { if } m<p+7, \\
& \beta-\gamma=\frac{8}{2} m+2 p+6 \geq 0 \text { if } m<\frac{6}{8} p+4
\end{aligned}
$$


If $2<p<9$ we have $0<2 p-2<\frac{4}{3} p+4<p+7$.

If $p=9$ the 3 limits coincide.

If $p>9$ we have

$$
0<p+7<\frac{4}{8} p+4<2 p-2 \text {, }
$$

and $3 p+3$ is of course always above all these limits.

8. Suppose first $p \geq 9$. We have to consider several cases.

Case 1.

$$
6<m<p+7 \text {. }
$$

Then $\gamma<\alpha<\beta$ and it is $\gamma$ that we must compare to $\kappa_{1}$ and $\kappa_{2}$. We have

$$
\begin{aligned}
\varphi(\gamma ; m, p) & =(3 m+p-9)[3 m+p-9-4 m-4 p+11] \\
& =(m-p)^{2}-5 m+p+6 .
\end{aligned}
$$

Let $m-p=9$, then $-p<9<7$ in the case considered. Also

$$
\varphi(\gamma ; m, p)=q^{2}-5 q-4 p+6=F(q) \text {. }
$$

If $q_{1}$ and $q_{2}$ are the roots of $F(q) \quad\left(q_{1}<q_{2}\right)$, we must locate them with respect to $-p$ and 7 . Since

also

$$
q_{1} q_{2}=-4 p+6<0 \quad q_{1}<0<q_{2},
$$

always, and

$$
F\left(-p^{\prime}\right)=p^{2}+p+6>0
$$

$$
F(7)=20-4 p<0
$$

in the case considered. Hence

$$
-p<q_{1}<0<7<q_{2},
$$

and $q_{1}$ is the only root in the interval considered. Accordingly if

$$
-p<q<q_{1}, \quad \varphi(\gamma)>0, \quad \gamma<\kappa_{1} ;
$$

but if

$$
q_{1}<q<7, \quad \varphi(\gamma)<0, \quad \kappa_{1}<\gamma .
$$

As

$$
q_{1}+p=\frac{5-\sqrt{16 p+1}}{2} \text { and }+p=\frac{2 p+5-\sqrt{16 p+1}}{2}
$$

we may conclude as follows:

$$
\begin{aligned}
& \text { If } m<\frac{2 p+5-\sqrt{16 p+1}}{2} \text { we must have } \kappa<\gamma \text {. } \\
& \text { If } m>\frac{2 p+5-\sqrt{16 p+1}}{2}, \kappa<\kappa_{1} \text {. }
\end{aligned}
$$

Case 2.

$$
p+7<m<2 p-2 \text {. }
$$


Then of the numbers $\alpha, \beta, \gamma, \alpha$ is the smallest and is therefore to be compared to $\kappa_{1}$ and $\kappa_{2}$. We have

$$
\begin{aligned}
\varphi(\alpha ; m, p)= & 2(m+p-1)[2(m+p-1)-4 m-4 p+11] \\
& +4\left[(m+p)^{2}-5 m-7 p+6\right] \\
= & -2[21 m+25 p-6]<0 .
\end{aligned}
$$

In this interval therefore we have again $\kappa_{1}<\alpha$ and the condition $\kappa<\kappa_{1}$.

Case 3.

$$
2 p-2<m \text {. }
$$

Then $\beta$ is smaller than either $\alpha$ or $\gamma$, and is to be compared to $\kappa_{1}$ and $\kappa_{2}$. We have:

$$
\begin{aligned}
\mathbf{4}(\beta ; m, p)= & 3(m+2 p-2)[3(m+2 p-2)-2(4(m+p)-11)] \\
& +16\left[(m+p)^{2}-5 m-7 p+6\right]=(m-2 p-1)^{2}-8 p+1 \\
= & (m-2 p-1-\sqrt{8 p+1})(m-2 p-1+\sqrt{8 p+1})<0
\end{aligned}
$$

in the interval considered, if $m<2 p+1+\sqrt{8 p+1}$, since here $2 p-2>2 p$ $+1-\sqrt{8 p+1}$ for $p>9$. Finally:

$$
\beta-\frac{1}{2}\left(\kappa_{1}+\kappa_{2}\right)=-\frac{1}{2} m-p+\frac{9}{2}<0 \text { for } p>9 \text {. }
$$

Therefore if $2 p-2<m<2 p+1+\sqrt{8 p+1}$, then $\kappa_{1}<\beta$ and therefore we must have $\kappa<\kappa_{1}$; while if $2 p+1+\sqrt{8 p+1}<m, \beta<\kappa_{1}$ and the condition is $\kappa<\beta$. This completes the discussion when $p \geq 9$.

9. Suppose now $2<p<9$.

Case 1.

$$
m<\frac{4}{2} p+4 .
$$

Then $\gamma$ is smaller than $\alpha$ or $\beta$, and is to be compared to $\kappa_{1}$. As in the preceding paragraph we find:

$$
\varphi(\gamma)=q^{2}-5 q-4 p+6=E(q) ;
$$

with $-p<q<\frac{1}{8} p+4$. Now

$$
F\left(\frac{1}{8} p+4\right)=\left(\frac{1}{8} p+4\right)\left(\frac{1}{8} p+4-5\right)-4 p+6=\frac{1}{8}\left(p^{2}-27 p+36\right) \text {. }
$$

The parenthesis has for roots 25.6 and 1.4 , hence here

and accordingly

$$
F\left(\frac{1}{8} p+4\right)<0,
$$

Hence if

$$
-p<q_{1}<0<\frac{1}{3} p+4<q_{2} \text {. }
$$

$$
m<\frac{2 p+5-\sqrt{16 p+1}}{2}<6, \quad \kappa<\gamma
$$


and if

$$
\frac{2 p+5-\sqrt{16 p+1}}{2}<m<\frac{4}{3} p+4, \quad \kappa<\kappa_{1} .
$$

Case 2.

$$
\frac{4}{3} p+4<m \text {. }
$$

Then $\beta$ is smaller than either $\alpha$ or $\gamma$.

We find as in section 8 that

$$
\varphi(\beta ; m, p)=(m-2 p-1+\sqrt{8 p+1})(m-2 p-1-\sqrt{8 p+1}),
$$

and it can be verified easily that

$$
2 p+1-\sqrt{8 p+1}<\frac{4}{8} p+4<2 p+1+\sqrt{8 p+1}
$$

when $p<9$. If then $\frac{4}{8} p+4<m<2 p+1+\sqrt{8 p+1}, \varphi(\beta)<0$ and $\kappa<\kappa$, is the condition; while if $2 p+1+\sqrt{8 p+1}<m$, then $\varphi(\beta)>0$ and we must have $\alpha<\beta<\kappa_{1}$.

We may remark that if $m>6$ and $p<1, m>2 p+1+\sqrt{8 p+1}$, and the condition $\alpha<\beta$ is also necessary. We shall see that it is also sufficient for the existence of the corresponding curves.

10. The preceding discussion can be tabulated as follows:

$$
\begin{gathered}
m\left|\begin{array}{c}
7 \cdots 2 p+1+\sqrt{8 p+1} \cdots+\infty \\
p<9 \mid \\
\kappa<\kappa_{1}, \quad \kappa<\frac{8}{2}(m+2 p-2)
\end{array}\right|, \\
m>9\left|\begin{array}{c}
\ldots \frac{2 p+5-\sqrt{16 p+1}}{2} \cdots 2 p+1+\sqrt{8 p+1} \cdots+\infty \\
\kappa<3 m+p-9, \quad \kappa<\kappa_{1}, \quad \kappa<\frac{8}{2}(m+2 p-2)
\end{array}\right| .
\end{gathered}
$$

The value of $\kappa_{1}$ is:

$$
\kappa_{1}=\frac{1}{2}[4(m+p)-11-\sqrt{-8 m+24 p+25}] .
$$

If any of the values given in the table exceeds $\frac{(m-1)(m-2)}{2}-p$ it simply means that all double points can $a$ priori become cusps for the system $(m, p)$ considered. It may be remarked that the only case in which the postulate of singularities affects the discussion is when

$$
7<m<\frac{2 p+5-\sqrt{16 p+1}}{2} \text { and } p>9,
$$

for if it is not admitted to be true, we have

$$
\gamma=3 m+p-9+\lambda(m, p),
$$

and it is only in this case that of the numbers $\alpha, \beta, \gamma$, the latter is the smallest. 
11. The following problem is also interesting: how many more double points or cusps can be added to a curve of order $m$ above $\delta$ double points and $\alpha$ cusps. The preceding discussion is of no avail inasmuch as $p$ is indefinite in this case. We shall see that this problem is suggested by the theory of surfaces.

We shall not carry the discussion into any details and shall merely state that for a concrete value of $m$, the best procedure is to plot curves giving $\alpha$ in terms of $p$ in

$$
\begin{gathered}
2(m+p-1)-\kappa=0, \\
3(m+2 p-2)-2 \kappa=0, \\
\varphi(\kappa ; m, p)=0, \quad 3 m+p-9-\kappa=0
\end{gathered}
$$

and then to find the region of the plane for which the left members are positive.

The same discussion can of course be applied to other singularities than cusps. Here however we are forcibly reminded of the fact that our discussion defines only upper limits which may or may not be reached. For example let us propose to determine the largest number of quadruple points that a seventhic can have. To have one quadruple point there must be 8 relations between its independent absolute invariants. The number of the latter is $7.10 / 2-8=27$, from which would follow that a seventhic may have as many as three quadruple points, but of course even if it has as many as two the curve will break up into a sextic and a straight line.

\section{Existence of the curves.}

12. While we have been unable to give positive existence proofs coverng the whole range of the tables of $\S 10$, we shall see that the range actually covered is quite extensive.

The following preliminary lemma is quite important. If for given $(m, p)$ all curves for which $\kappa<\kappa_{0}$ exist, the same holds for the curves having the characters $m, p^{\prime}, \kappa^{\prime}$ such that $p^{\prime} \geq p, \kappa^{\prime}<\kappa_{0}$. For we may take the general equation of curves of the $m$ th order, and make it represent a curve with characters $(m, p, \kappa)$. As coefficients and the coördinates of these singular points will have to satisfy a certain system $E$ composed of a certain number of equations and inequalities. If $p^{\prime} \geq p, \kappa^{\prime}<\kappa$, it is clear that the system $E^{\prime}$ corresponding to $\left(m, p^{\prime}, \kappa^{\prime}\right)$ is contained in the system $E$, by which we express the fact that the equations and inequalities of $E^{\prime}$ are respectively among the equations and inequalities of $E$. Any solution of $E$ will be a solution of $E^{\prime}$, but not the only one, for then $E$ could be reduced to a simpler system, in which case some of the double points or cusps of the curve corresponding to $(m, p, \kappa)$ would be the consequence of others, but this is against the supposition that for the values $(m, p)$, curves exist for all values of $\kappa$ inferior to $\kappa_{0}$, and also 
against the fact that double points are independent $(\S 3)$. Our lemma is thus proved.

13. We shall now proceed to show that a curve with characters $(m, p, \kappa)$, for which the table in $\S 10$ gives the condition $\kappa<\frac{8}{2}(m+2 p-2)$, exists if $\kappa$ is inferior to this limit. Referring to the tables this means that if

$$
m \geq 2 p+1+\sqrt{8 p+1}
$$

and the curve has positive characters, its existence can be proved. For then

$$
m>2(p-1)+1+\sqrt{8(p-1)+1}
$$

and therefore the table will give for the curves having the characters $(m, p-1, \kappa)$ the condition:

$$
\kappa<\frac{3}{2}[m+2(p-1)-2] \text {. }
$$

Suppose now that the proposition is proved for the curve corresponding to $(m, p-1, \kappa)$, then by our lemma the curve corresponding to $(m, p, \kappa)$ can have as many as $\left[\frac{8}{2}[(m+2(p-1)-2)]\right]$ cusps. Suppose that

then will

$$
\kappa=\left[\frac{8}{2} m\right]+3(p-2)+h ; \text { and } 1<h<3 \text {, }
$$

$$
\begin{aligned}
& h=2(m+p-1)-\left[\frac{3}{2} m\right]+3(p-2)+h=\frac{m+e}{2}-(p+h-4), \\
& i=3(m+2 p-2)-2\left[\frac{3}{2} m\right]-6(p-2)-2 h=e+2(3-h),
\end{aligned}
$$

with $e=0$ or 1 according as $m$ is odd or even. Now $(n, p, i)$ are the characters of the reiprocal of the curve considered. It is clear that $i<5$, hence either $n<6$, - and then since the reciprocal has positive characters its existence is certain; or $n>6$, and then our lemma applies (since a curve having characters $(n, 0, \kappa)$ can have as many as $n-2$ cusps $(\$ 4)$, and $n-2 \geq 5$ here), it follows that a curve of order $n$ and genus $p$ can certainly have as many as 5 cusps when $n>6$. In both cases the reciprocal exists certainly, and the same holds for the curve with characters $(m, p, \kappa)$; this proves the proposition considered, as soon as it is proved for $p=0$. This however has been done by Veronese (loc. cit., $\$ 1$ ), and we shall also give a proof later.

Let us now find the limiting values of $p, \alpha$ for which the theorem holds. We must suppose $m$ given and solve the equation

for $p$. It includes:

$$
m \geq 2 p+1+\sqrt{8 p+1}
$$

$$
m>2 p+1 \quad \text { or } \quad p<\frac{m-1}{2}
$$


which remark after a simple calculation shows that

then

$$
p<\frac{m+3-\sqrt{3(2 m+3)}}{2} \text {; }
$$

$$
\beta=\frac{8}{2} m+3 p-3=3\left(m+\frac{1}{2}\right)-\frac{8}{2} \sqrt{3(2 m+3)},
$$

and this is the highest value of $\kappa$, the existence of which is certain. Remarking that the proposition can be applied when

$$
n \geq 2 p+1+\sqrt{8 p+1},
$$

we obtain a new extension of the limits found. This will occur if

$$
2(m+p-1)-\kappa \geq 2 p+1+\sqrt{8 p+1},
$$

or if $0<\kappa<2 m-3-\sqrt{8 p+1}$, that is, for given $(m, p)$ a curve can have as many as $1,2, \cdots,(2 m-3-\sqrt{8 p+1})$ cusps.

14. There is a class of special cuspidal curves for which an equation can be given readily. Let $X=0, Y=0$ represent two straight lines not passing through any of the vertices of the triangle of reference. Then

$$
x y z(a X+b X)^{m-3}+X^{m}=0
$$

is the equation of a curve having a contact of order $(m-1)$ at the intersection of $X=0$ with the sides of the triangle of reference and also a point of multiplicity $(m-3)$ with as many coincident tangents at the intersection of $X=0$ with $Y=0$. This multiple point is equivalent to $(m-4)$ cusps and a certain number of double points. If we apply now the transformation:

we obtain

$$
x^{\prime} x=y^{\prime} y=z^{\prime} z
$$

$$
\frac{1}{x^{\prime} y^{\prime} z^{\prime}}\left(\frac{a^{\prime}}{x^{\prime}}+\frac{b^{\prime}}{y^{\prime}}+\frac{c^{\prime}}{z^{\prime}}\right)^{m-8}+\left(\frac{d^{\prime}}{x^{\prime}}+\frac{e^{\prime}}{y^{\prime}}+\frac{f^{\prime}}{z^{\prime}}\right)^{m}=0 \text {. }
$$

This is a curve of order $m^{\prime}=2 m$, having four points of multiplicity $m$, $m, m, m-3$ respectively at each of which the tangents all coincide. The equivalent number of cusps is $3(m-1)+m-4=4 m-7=2 m^{\prime}-7$. To obtain curves of odd order consider:

$$
x y z(a X+b Y)^{m-3}+X^{m-1}(X+c x)=0 .
$$

The same tranformation will give, if $X+c x \equiv d y+e z$, the following equation:

$$
\left(\frac{a^{\prime}}{x^{\prime}}+\frac{b^{\prime}}{y^{\prime}}+\frac{c^{\prime}}{z^{\prime}}\right)^{m-3}+x^{\prime}\left(-\frac{c}{x^{\prime}}+\frac{d}{y^{\prime}}+\frac{e}{z^{\prime}}\right)^{m-1}\left(d z^{\prime}+e y^{\prime}\right)=0,
$$

which represents a curve of order $m^{\prime}=2 m-1$, with four points of multi- 
plicity $m-1, m-1, m, m-3$, respectively, at each of which all tangents are coincident. The equivalent number of cusps is: $2(m-2)+m-1$ $+m-4=4 m-9=2\left(2^{m}-1\right)-7=2 m^{\prime}-7$ again. In both cases

$$
p=\frac{(m-1)(m-2)}{2}-\frac{(m-3)(m-4)}{2}=2 m-5=2\left[\frac{m^{\prime}+1}{2}\right]-5 .
$$

If instead of $(a X+b Y)^{m-3}$ we had taken $(a X+b Y)^{m-3-h} \varphi_{h}(x, y, z)$, we should have obtained the following two equations:

$$
\begin{aligned}
& \frac{1}{x^{\prime} y^{\prime} z^{\prime}}\left(\frac{a^{\prime}}{x^{\prime}}+\frac{b^{\prime}}{y^{\prime}}+\frac{c^{\prime}}{z^{\prime}}\right)^{m-3-h} \varphi_{h}\left(\frac{1}{x^{\prime}}, \frac{1}{y^{\prime}}, \frac{1}{z^{\prime}}\right)+\left(\frac{d^{\prime}}{x^{\prime}}+\frac{e^{\prime}}{y^{\prime}}+\frac{f^{\prime}}{z^{\prime}}\right)^{\frac{m^{\prime}}{2}}=0 \\
& \frac{1}{x^{\prime} y^{\prime}} \overline{z^{\prime}}\left(\frac{a^{\prime}}{x^{\prime}}+\frac{b^{\prime}}{y^{\prime}}+\frac{1^{\prime}}{z^{\prime}}\right)^{\frac{m-5-h}{2}} \varphi_{h}\left(\frac{1}{x^{\prime}}, \frac{1}{y^{\prime}}, \frac{1}{z^{\prime}}\right)+\left(\frac{d^{\prime}}{x^{\prime}}+\frac{e^{\prime}}{y^{\prime}}+\frac{f^{\prime}}{z^{\prime}}\right)^{\frac{m^{\prime}-1}{2}}\left(\frac{e^{\prime}}{y^{\prime}}+\frac{f^{\prime}}{z^{\prime}}\right)=0 \\
& \text { ( } m^{\prime} \text { odd), }
\end{aligned}
$$

representing curves having $\left(2 m^{\prime}-h-7\right)$ cusps, with

$$
h<\left\lfloor\frac{m^{\prime}+1}{2}\right]-3 \text {. }
$$

We merely indicate here that by making the curve $\varphi_{h}(x, y, z)=0$ have a certain number of multiple points on $d x+c y+f z=0$ the above curves can be made to have a genus varying within a certain range. It would not be uninteresting to find whether $p$ can be made to have the same value when $h$ changes; it is no doubt possible within certain limits.

15. We have already stated that curves of order inferior to 6 are well known to exist when their characteristics are positive. The same may be stated for the case $m=6$, but as no one to the best of our knowledge has considered them under this light, and as it is important for what follows, we shall briefly consider sextics, and show the existence of any sextic with positive characters.

(1) Rational sextics. The proof of their existence follows the same lines as the one which will be given subsequently. A rational sextic has at most $3(6-2)=6$ cusps. A sexticuspidal sextic has for reciprocal a quartic, a quinticuspidal sextic has for reciprocal a quintic, so that their existence is certain. Next, the equation

$$
z(x-\alpha y)^{h}\left(x-\beta_{\mathfrak{i}} y\right) \cdots\left(x-\beta_{5-h} y\right)+\varphi_{6}(x, y)=0
$$

represents a rational sextic having a singularity equivalent to $5-h$ cusps and $5+h$ double points.

(2) Elliptic sextics. In this case all double points may become cusps. The sextics with $9,8,7$ cusps are inverse of a cubic, quartic, quintic, respectively. If we consider next the triangle $A B C$ formed by three inflexional tangents to 
a cubic, and if we apply to the curve the quadratic transformation of $\S 14$ we obtain a sextic having three triple points with coincident tangents. Each of these triple points is equivalent to 2 cusps, hence we have obtained a particular case of the curve $(6,1,6)$. If $B C$ were merely tangent to the cubic or absolutely arbitrary we should have particular cases of sextics with 5 or 4 cusps, and genus one. By modifying $A B$ and $A C$ we should obtain the remaining cases.

(3) Genus superior to one. The existence of all sextics with positive characters, for $p>1$, follows from the proposition given in $\S 12$, and if necessary their existence could be shown by the methods used for rational and elliptic sextics, but we will not insist.

Existence of the curves of genus 1, 2, 3, 4 and 5.

16. Rational curres. We have given $(\$ 4)$ the equation of these curves when $\kappa<m-2$. We shall now show the existence of all rational curves with less than $\left[{ }_{2}^{3}(m-2)\right]$ cusps. Suppose that

Then we find:

$$
\kappa=(m-2)+g, \quad g<1(m-2) .
$$

$$
\begin{aligned}
n & =2(m-1)-\kappa=m-g>0, \\
i & =3(m-2)-2(m-2+g)=m-2-2 g \\
& =\dot{m}-g-(g+2)=n-g-2<n-2 .
\end{aligned}
$$

If we consider then the equation:

$$
z(x-a y)^{m-1-2 g} \varphi_{0}(x, y)+\varphi_{m-g}(x, y)=0,
$$

it represents a curve the reciprocal of which has $m-2+g$ cusps, hence

$$
w(u-a v)^{m-1-2 \sigma} \varphi_{o}(u, v)+\varphi_{m-o}(u, v)=0
$$

is the tangential equation of a rational $m$ thic with $(m-2+g)$ cusps $\left(g<\left[\frac{1}{2}(m-2)\right]\right)$.

It is interesting here to recall Veronese's * method of establishing the same result. More generally he showed that the curve having the characters $(m, p, \kappa)$ exists if $\kappa<\frac{3}{2}[m-p-2]$. If $p=0$ we obtain $\frac{3}{2}[m-2]$. He considers the $C_{m}^{m-p}$ of order $m$ and genus $p$, in $(m-p)$ fold space, and shows that there can always be found an $(m-p-3)$ fold flat meeting $\frac{3}{2}\left[m-p-2\right.$ ] (or less) straight lines, in particular tangents of $C_{m}^{n-p}$, in the space considered. By projecting then from the $(m-p-3)$ flat into a plane, a $C_{m}^{2}$ is obtained with $\frac{3}{2}(m-p-2)$ cusps or less. There is little doubt that by extending the method of $\S 14$ as indicated at the end, we could obtain equations or curves with any number of cusps up to $2 m-7$ and assigned

\footnotetext{
- Loc. cit.
} 
deficiency - within certain limits perhaps - and this number of cusps is much higher than the limit given by Veronese. It may be stated that Veronese's proof shows that any curve of order $m$ and genus $p$ can be birationally transformed into another of the same order in such a way that $\frac{8}{2}(m-p-2)$ of its points become cusps of the transformed curve.

In his memoir Veronese states also that the extension of his method to the case $p \neq 0$, depends upon an investigation of the number of tangents of a $C_{m}^{m-p}$ in an $(m-p)$ fold space that can meet an $(m-p-3)$-flat of this space. Our problem is then so to dispose of the moduli (in Riemann's sense) of $C_{m}^{m-p}$ that it have a maximum number of tangents meeting an $(m-p-3)$ flat, and in this disposing of the moduli lies the difficulty.

Elliptic curves. The lowest value of $m$ for which the theorem of $\$ 13$ applies in this case is: $m=2.1+1+\sqrt{8+1}=6$ and by $\S 15$ follows the certainty of the existence of all elliptic curves with positive or zero characters.

17. Curves of genus two. From $\$ 13$ follows their existence when

$$
m>2.2+1+\sqrt{8.2+1}=9,1 \text { or } 10 \text {. }
$$

For $m=7,8,9$, a direct examination is necessary.

Case 1. $m=7$. We must have $\kappa \gtrless \kappa_{1}$ and we find

$$
\kappa_{1}=\left[\frac{1}{2}(4(7+2)-11-\sqrt{-8.7+24.2+25)}]=10 .\right.
$$

But for $m=7, p=1$, the limit is $x<\left[\frac{8}{2}[7+2-2]\right]=10$. So that we know that all curves for which $m=7, p=1, \kappa<10$, exist, hence ( $\S 12$ ) the same is true for the curves such that $m=7, p=2, \kappa<10$.

Case 2. $m=8$. We find here $\kappa_{1}=13$, so that we must have $\kappa<13$. The limit for the case $m=8, p=1$ is $\left[\frac{8}{2}(8+2 .-2)\right]=12$. Hence the only curve to be considered is the one for which $k=13$. But its-reciprocal is found to be a quintic, and therefore exists necessarily, so that the same is true for the curve in question.

Case 3. $m=9$. In this case $\kappa_{1}=16$. For $p=1, m=9$ it is found that $\kappa<13$, while if $\kappa=14,15,16$, the curve is the reciprocal of curves of order $6,5,4$, hence its existence is certain.

We have therefore shown the existence of all curves of genus two with positive characters.

18. Curves of genus three. From $\$ 13$ follows their existence when $m \geq 2.3$ $+1+\sqrt{8.3+1}=12$. We must therefore consider separately curves of order $7,8,9,10,11$.

(1) $m=7$. In this case $\kappa_{1}=11$. When $p=2$ we know that $\kappa$ can have any value inferior to 10 , and we must therefore merely prove the existence of the curve for which $k=11$. The characters of the reciprocal curve are the same, and none of the other methods of this paper succeed with this curve, the existence of which we have not succeeded in proving as yet. 
(2) $m=8$. Here $\kappa_{1}=13$, the same limit as for $p=2$, hence by $\S 12$ we know that $\kappa$ can have all values smaller than 13 .

(3) $m=9$. Here $\kappa_{1}=16$, and the limit is again the same as for $p=2$.

(4) $m=10$. Again $\kappa_{1}=18=\beta(10,2)$.

(5) $m=11$. Here $\kappa_{1}=21$, and for $p=2$ we must have $\kappa \overline{<} \beta(11,2)=19$. Hence we have to consider only the cases $\kappa=20,21$; but then the class being less than 9 , we are reduced to a preceding case. We can therefore conclude to the existence of all curves of genus three with positive characters, the curve $(7,3,11)$ being excepted.

19. Without further detailed discussion, we shall merely state that this method can be used to prove the existence of all curves of genus four or five, with the exception of the following two:

$$
\begin{array}{lll}
m=8, & p=4, & \kappa=14 ; \\
m=8, & p=5, & \kappa=16 ;
\end{array}
$$

as they have the class equal to the order. Moreover it can be easily shown that when $m$ and $p$ both increase there is always a value of $m$ limiting the range of application of the method for a given value of $p$. More precisely, if

$$
m<\frac{\sqrt{24 p-39}+9}{2}
$$

some of the curves whose existence is to be proved will have reciprocals of order higher than their own, or equal to it.

\section{Singularities of surfaces and higher varieties.}

20. Surfaces and varieties of $(n-1)$ dimensions in $n$-space can be treated like plane curves. It can be shown for them also that they have in general $\frac{m(m-1) \cdots(m-n+1)}{n !}-(n+1)^{2}$ absolute invariants, unless they are invariant under a continuous projective group of transformations. Hence * by a well-known theorem: When a surface of order $m$ has a positive numerical genus $p_{n}$, it has exactly

$$
\frac{(m+1)(m+2)(m+3)}{6}-16
$$

absolute invariants. For a surface invariant under any group of transformations can be birationally transformed into a surface, for which $p_{n}<0$. In particular a surface of order greater than 3 and having no other singularities than nodes or binodes satisfies the above condition. Another criterion

\footnotetext{
- Picard et Smart : Traite des fonctions algcbriques de deux variables, vol. 2, page 520.
} 
which we shall not develop here but which results easily from a short paper of Aronhold * states that: If the first polars of $m$ independent points have in common a system of $(m+1)$ points not situated on an arbitrary first polar and forming a true $(m+1)$-edron, then the variety is not invariant under any projective group and the number of absolute invariants is as given above.

To find the a priori possible surfaces or varieties we discuss the tangent hypercone. In case of a surface, the section of the tangent cone will have a minimum number of double points and cusps, and the question will be reduced to the one outlined in $\S 11$. If $\delta$ is the number of quadratic nodes, $\kappa$ the number of ordinary quadratic binodes, and if the surface has no other singular points or curves than these, then we have the condition

$$
\delta+2 \kappa<\frac{(m+1)(m+2)(m+3)}{6}-16+\lambda
$$

with $\lambda=0$ if the extended postulate of singularities is admitted.

21. Let us outline briefly the discussion for quartic surfaces. In this case there are 5.6.7/6-16=19 absolute invariants. The tangent cone is of order 12 , and has $\frac{1}{2} \cdot 4.3 .2 .1 .+\delta=12+\delta$ double edges and 4.3.2 $+\kappa$ $=24+\kappa$ cuspidal edges.

Let $n$ be the class of this cone, $i$ and $\tau$ the number of its inflexional and bitangential planes. We then have:

$$
\begin{gathered}
n=12.11-2(\delta+12)-3(\kappa+24), \\
i=3.12 .10-6(\delta+12)-8(\kappa+24), \\
2 \tau=2(\delta+12)+(n-12)(n+12-9), \\
\delta+2 \kappa<19 .
\end{gathered}
$$

If instead of $p$, we introduce $\delta+\kappa=\lambda$ and write the conditions that the characters be positive or zero, we obtain:

$$
\begin{gathered}
36-2 \lambda-\kappa \geq 0, \\
48-3 \lambda-\kappa \geq 0 \\
\lambda+\kappa<19, \\
2 \tau=(\kappa+2 \lambda)^{2}-124 \kappa-65 \lambda+960 \geq 0 ;
\end{gathered}
$$

and the discussion is best carried out by means of diagrams, which we will omit here. The following results worthy of notice are then obtained:

(1) The number of nodes cannot exceed 16 - a well-known fact.

(2) If the number of nodes is inferior to 8 they can all be binodes.

\footnotetext{
* Fundamentalsatz der Ineariantentheorie. C r e 11 e's J o u r n a l, vol. 69 (1868), p. 185.
} 
(3) There cannot be any binodes when the total number of nodes exceeds 14.

It is found by a similar discussion that a quintic surface cannot haye more than 34 nodes, and that if it has one binode the number of nodes is not superior to 33 , while the total number of binodes can never exceed 20 .

22. This discussion applied to cubic varieties gives several interesting results. In the first place the maximum number of nodes as shown by the postulate of singularities, viz. 10, can be reached, and we have then the Segre variety. Next, this postulate gives 5 as the maximum number of nodes of the second species, and the writer has shown elsewhere the existence of the corresponding variety.*

In conclusion our thanks are due to Professor W. E. Story for suggesting the investigations of this paper.

Worchster, Mass.,

June 15, 1911.

* Bulletin of the American Mathematical Societv. vol. 18 (1912), p. 384. 